\title{
Identification of key genes associated with progression and prognosis for Lung Squamous Cell Carcinoma
}

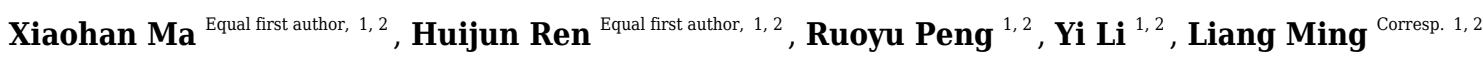 \\ ${ }^{1}$ Department of Clinical Laboratory, the First Affiliated Hospital of Zhengzhou University, Zhengzhou, Henan, China \\ 2 Key Clinical Laboratory of Henan Province, Zhengzhou, Henan, China \\ Corresponding Author: Liang Ming \\ Email address: mingliang_2015@sina.com
}

Background. Lung squamous cell carcinoma (LUSC) is a major subtype of lung cancer with limited therapeutic options and poor clinical prognosis.

Methods. Three datasets (GSE19188, GSE33532, and GSE33479) were obtained from the gene expression omnibus (GEO) database. Differentially expressed genes (DEGs) between LUSC and normal tissues were identified by GEO2R, and functional analysis was employed using DAVID online tool. Proteinprotein interaction (PPI) and hub genes were identified via Search Tool for the Retrieval of Interacting Genes (STRING) and Cytoscape software. Hub genes were further validated in TCGA database. Subsequently, survival analysis was performed using the Kapla-Meier curve and Cox progression analysis. Based on univariate and multivariate Cox progression analysis , a gene signature was established to predict overall survival. Receiver operating characteristic (ROC) curve was used to evaluate the prognostic value of the model.

Results. A total of 116 up-regulated genes and 84 down-regulated genes were identified. These DEGs were mainly enriched in the two pathways: cell cycle and p53 signaling way. According to the degree of protein nodes in the PPI network, ten hub genes were identified. The mRNA expression levels of the ten hub genes in LUSC were also significantly up-regulated in TCGA database. Furthermore, a novel sevengene signature (FLRT3, PPP2R2C, MMP3, MMP12, CAPN8, FILIP1 and SPP1) from the DEGs was constructed and acted as a significant and independent prognostic signature for LUSC.

Conclusions. The ten hub genes might be tightly correlated with LUSC progression. The seven-gene signature might be an independent biomarker with a significant predictive value in LUSC overall survival. 


\section{Identification of key genes associated with progression and 2 prognosis for Lung Squamous Cell Carcinoma}

4 Xiaohan $\mathrm{Ma}^{* 1,2}$, Huijun Ren ${ }^{* 1,2}$, Ruoyu Peng ${ }^{1,2}, \mathrm{Yi} \mathrm{Li}^{1,2}$, Liang Ming ${ }^{1,2}$

$5{ }^{1}$ Department of Clinical Laboratory, the First Affiliated Hospital of Zhengzhou University, Zhengzhou,

6 Henan, China

$7 \quad 2$ Key Clinical Laboratory of Henan Province, Zhengzhou, Henan, China

$8 *$ These authors are co-first authors on this work.

13 Corresponding Author: Liang Ming

14 No. 1 Jianshe Road, Zhengzhou, Henan, 450052, China

15 Email address: mingliang_2015@sina.com 
16 Abstract

17 Background. Lung squamous cell carcinoma (LUSC) is a major subtype of lung cancer with

18 limited therapeutic options and poor clinical prognosis.

19 Methods. Three datasets (GSE19188, GSE33532, and GSE33479) were obtained from the gene 20 expression omnibus (GEO) database. Differentially expressed genes (DEGs) between LUSC and 21 normal tissues were identified by GEO2R, and functional analysis was employed using DAVID 22 online tool. Protein-protein interaction (PPI) and hub genes were identified via Search Tool for 23 the Retrieval of Interacting Genes (STRING) and Cytoscape software. Hub genes were further 24 validated in TCGA database. Subsequently, survival analysis was performed using the Kapla25 Meier curve and Cox progression analysis. Based on univariate and multivariate Cox progression analysis, a gene signature was established to predict overall survival. Receiver operating 27 characteristic (ROC) curve was used to evaluate the prognostic value of the model.

Results. A total of 116 up-regulated genes and 84 down-regulated genes were identified. These DEGs were mainly enriched in the two pathways: cell cycle and p53 signaling way. According to the degree of protein nodes in the PPI network, ten hub genes were identified. The mRNA expression levels of the ten hub genes in LUSC were also significantly up-regulated in TCGA database. Furthermore, a novel seven-gene signature (FLRT3, PPP2R2C, MMP3, MMP12, CAPN8, FILIP1 and SPP1) from the DEGs was constructed and acted as a significant and independent prognostic signature for LUSC.

Conclusions. The ten hub genes might be tightly correlated with LUSC progression. The sevengene signature might be an independent biomarker with a significant predictive value in LUSC overall survival.

\section{Introduction}

Lung cancer, as a highly malignant cancer, is still a common cause for healthy issues worldwide. With approximately 1.8 million deaths in 2018 (Bray et al., 2018), lung cancer has been ranked in top 2 in China and possessed the highest mortality rate (Sun et al., 2018). Among all the lung cancer types, non-small cell lung cancer (NSCLC) accounts for over $85 \%$ of total cases. Lung squamous cell carcinoma (LUSC) is the second most frequent subtype of NSCLC, accounting for about 40\% of NSCLC (Chen et al., 2014), and its therapy and prognosis are still facing huge challenges. Nowadays, surgery and adjuvant chemotherapy are the standard $-$ 
47 treatment for stage I and II of NSCLC, however, molecular analysis is the key to select a first-

48 line therapy for advanced cancer (Kuo et al., 2019). Large numbers of gene mutations been reported to be served as specific biomarkers for diagnosis, treatment and prognosis for LUAD (lung adenocarcinoma) (Calvayrac et al., 2017), such as bevacizumab against VEGF. Although complex genomic alterations were found in LUSC, which were different from LUAD, there were no available and specific targeted agents for LUSC until now (Hirsch et al., 2017). Despite great progress made in combined therapies, the prognosis of LUSC is still dismal. Hence, there is an imperative and urgent need for identify key molecules for therapy and prognosis of LUSC.

Recently, high throughput sequencing and microarray technologies have been widely used to investigate relationship between diverse diseases and key molecules, including genes, miRNAs, long non-coding RNAs (lncRNAs), and circRNAs. Yeo et al (Yeo et al., 2017) proved that programmed cell death 1 was over-expressed in LUSC and could be useful for the prediction of poor prognosis. Hou et al identified 95 up-regulated and 749 down-regulated lncRNAs in response to cisplatin chemo, which indicate that dysregulated lncRNAs are related with therapy and are prognostic markers in LUSC (Hou et al., 2014). Several other key molecules were also found involved in the development, diagnosis, and prognosis of LUSC, such as circRNA_103827, circRNA_000122 (Xu et al., 2018), peroxiredoxin 4 (Hwang et al., 2015), AURKA, BIRC5, LINC00094 (Li et al., 2017), and so on. The dysregulation of these mentioned molecules is associated with the progression and prognosis of LUSC, however, limited samples and significant variability among different projects reduce the credibility of these obtained results.

In order to search for promising key genes associated with the progression and prognosis of LUSC, differentially expressed genes (DEGs) in LUSC were identified using three microarray datasets from the Gene Expression Omnibus (GEO) database. Subsequently, ten hub genes were identified by Gene Ontology (GO), Kyoto Encyclopedia of Genes and Genomes (KEGG) enrichment analysis and protein-protein interaction (PPI) network, which were involved in cell cycle and p53 pathway in LUSC. The expression of the ten hub genes was validated in TCGA database. In addition, a novel seven-gene signature was established to predict effectively overall survival in LUSC.

\section{6}

\section{Materials \& Methods}




\section{Data collection}

The mRNA expression profiles and corresponding clinical information were acquired from the GEO database (https://www.ncbi.nlm.nih.gov/geo/) and the Cancer Genome Atlas (TCGA) database (https://portal.gdc.cancer.gov). With searching for "lung squamous cell carcinoma", a total of 4627 series about LUSC were searched from the GEO database. After a careful review, three gene expression profiles (GSE19188, GSE33532, and GSE33479) were collected. The former two databases were both based on GPL570 platform ([HG-U133_Plus_2] Affymetrix Human Genome U133 Plus 2.0 Array), and GSE33479 was based on GPL6480 platform (Agilent-014850 Whole Human Genome Microarray 4x44K G4112F). 57 LUSC tissue samples and 112 normal lung tissue samples were collected from the three GEO datasets. 551 samples were collected from TCGA database, containing 502 LUSC tissues samples and 49 normal tissue samples.

\section{Data pre-processing and identification of DEGs}

In this study, the GEO2R (https://www.ncbi.nlm.nih.gov/geo/geo2r/), an interactive web tool used to compare two groups of samples according to GEO series, was applied to detect the DEGs between 57 LUSC samples and 112 normal samples. The adjusted P-value $<0.05$ and

$\mid \log 2$ (fold change) $\mid \geq 2.0$ were regarded as the cutoff criteria to select the DEGs. Then the DEGs obtained from different GSE datasets were further identified using the Venn diagram web tool (bioinformatics.psb.ugent.be/ webtools/Venn/).

\section{Functional analysis and PPI network construction of DEGs}

Gene Ontology (GO) and Kyoto Encyclopedia of Genes and Genomes (KEGG) analysis of DEGs were performed using the Database for Annotation, Visualization, and Integrated Discovery (DAVID) (https://david.ncifcrf.gov/). The adjusted $P$-value $<0.05$ was considered as statistically significant. In order to evaluate protein-protein interactions of DEGs, Cytoscape software and the Search Tool for the Retrieval of Interacting Genes (STRING) (https://string$\underline{\mathrm{db} .0 r g /)}$ were used to analyze PPI relationship, and PPI pairs with a combined score $>0.4$ were extracted. A cytoscape plugin, cytoHubba, was utilized to calculate the degree of protein nodes, and the top rank ten genes were selected as hub genes.

\section{Hub genes validation and analysis}

The mRNA expression profiles in 502 LUSC samples and 49 normal samples from TCGA database were analyzed by the "limma" R package. The statistical analysis was performed using 
109 the Wilcox test, and adjusted $\mathrm{P}<0.05$ and $\mid \log 2$ (fold change) $\mid \geq 2.0$ were selected as the cutoff

110 criteria. Subsequently, the expression levels of hub genes were validated in TCGA database.

111 Besides, the expression levels of the ten hub genes in LUSC were compared with other NSCLC

112 histologic subtypes using the Oncomine database (www.oncomine.org).

113 The Cox proportional hazards regression model was used for overall survival (OS) analysis

114 among the ten hub genes. Besides, Kaplan-Meier Plotter (http://kmplot.com/) was used to assess

115 the effect of the hub genes on LUSC prognosis. The option "only JetSet best probe set" was

116 selected for probes of genes, and only the LUSC patients were selected for analysis. At last, there

117 were 524 LUSC patients for OS analysis, 141 LUSC patients for FP analysis, and 20 LUSC

118 patients for PPS analysis in the Kaplan-Meier Plotter. $\mathrm{P}<0.05$ was regarded as statistically

119 significant. Then the genes with prognostic values were analyzed to identify their associations

120 with tumor grade using the Oncomine database.

\section{Survival analysis}

122 In order to identify the potential prognostic values of the DEGs in LUSC, univariate and 123 multivariate Cox regression were conducted using the $\mathrm{R}$ package. The clinical information of 124 LUSC patients was downloaded from TCGA database, and 488 LUSC patients were used for 125 analysis after removing the patients (14 of 502) with incomplete clinical data. Only 187 of 200

126 DEGs could be found and validated in TCGA database, and they were used for the univariate

127 Cox regression analysis. The genes associated with overall survival $(\mathrm{P}<0.05)$ were subjected to

128 the multivariate Cox proportional hazards model to establish a robust gene prognostic signature

129 for LUSC. The LUSC patients were further grouped into "high-risk" and "low-risk" based on the 130 median risk score. What's more, a receiver operating characteristic (ROC) curve was constructed 131 to evaluate the predictive accuracy of the gene signature by using the R package "survival ROC". 132 Besides, the gene signature and clinicopathological parameters (age, gender, tumor stage, T/N/M 133 status) were submitted to Cox regression analysis to identify independent factors for OS in 134 LUSC patients.

\section{Results}

\section{Identification of DEGs in LUSC}

All the mRNA expression profiles of GSE19188, GSE33479, and GSE33532 were provided 
140 1A). From GSE33479, 1153 DEGs were identified, including 499 up-regulated genes and 654 141 down-regulated genes (Fig. 1B). From GSE33532, 1375 DEGs including 569 up-regulated genes 142 and 806 down-regulated genes were identified (Fig. 1C). All the DEGs were identified by 143 comparing LUSC samples and normal lung samples. Subsequently, the results from the three 144 studies were analyzed using the Venn diagram tool, and 116 up-regulated genes and 84 down145 regulated genes were identified (Fig. 1D-E, Table S4).

146 Functional analysis and PPI network construction

147 GO and KEGG pathway enrichment analysis of DEGs were carried out to explore the 148 biological functions of 200 DEGs (Table 1). The enriched GO terms of DEGs were classified 149 into three categories: molecular functions (MF), cellular components (CC), and biological 150 processes (BP). As shown in Fig. 2, the DEGs were mainly enriched in "mitotic nuclear 151 division", "cell division", and "sister chromatid cohesion" in the BP category. Within the CC 152 category, "condensed chromosome kinetochore", "chromosome", and "midbody" were 153 predominant. The results of KEGG analysis exhibited that the DEGs were mainly involved in 154 "Cell cycle" and "p53 signaling pathway".

155 PPI analysis could reflect the molecular mechanisms of cancer physiological and 156 pathological changes. The PPI network of common DEGs was constructed using the STRING 157 V11.0 tool and Cytoscape software (Fig. 3), which containing 199 nodes and 2363 edges.

158 Subsequently, the top ten genes with high node degree were selected as the hub genes for further 159 analysis (Fig. 4). Results showed that the top ten hub genes were significantly up-regulated in LUSC tissues. Among them, cyclin-dependent kinases 1 (CDK1) was the most significant gene with connectivity degree $=57$, followed by BUB1 mitotic checkpoint serine/threonine kinase (BUB1; degree=48), cyclin B1 (CCNB2; degree=48), cyclin A2 (CCNA2; degree=46), kinesin family member 2C (KIF2C; degree=41), aurora kinase B (AURKB; degree=41), kinesin family member 11 (KIF11; degree=41), mitotic arrest deficient 2 like 1 (MAD2L1; degree=41), DNA topoisomerase II alpha (TOP2A; degree=40), and DLG associated protein 5 (DLGAP5; degree $=39$ ).

167

168

169

170

\section{Hub genes validation and analysis}

The expression of the ten hub genes was validated in TCGA database, which was consistent with the results from the GEO database (Fig. 5). The Analysis indicated that their expression levels in LUSC were significantly higher than those in other NSCLC (Fig. 6). Cox regression 
171 model was used to analyze the ten hub genes, however, none of them were associated with OS,

172 and no significant gene signature could be established to predict OS in LUSC patients. Kaplan-

173 Meier Plotter showed that the up-regulated CCNA2, KIF11, MAD2L1, and DLGAP5 were all

174 related to worse first-progression survival (FP) in LUSC patients (Fig. 7, A-D). Only the up-

175 regulation of KIF2C was associated with unfavorable post-progression survival (PPS) in LUSC

176 patients (Fig. 7E). Among the five hub genes with prognostic values genes, four (CCNA2,

177 DLGAP5, MAD2L1, and KIF2C) were associated with LUSC tumor grade (Fig. 8).

178 Cox progression analysis and construction of prognostic signature

179 Only 187 of 200 DEGs were found and validated in TCGA database. Then the 187 DEGs

180 were used for the univariate Cox regression analysis, and 23 of 187 DEGs were significantly

181 associated with the OS $(\mathrm{P}<0.05)$, as shown in Table S5. Subsequently, a seven-gene prognostic

182 signature was established by the multivariate Cox regression analysis (Table 2). Risk Score =

$1830.0341 \times$ expression of FLRT3 $+0.0339 \times$ expression of PPP2R2C $+0.0058 \times$ expression of

$184 \mathrm{MMP} 3+0.0031 \times$ expression of MMP12 $+0.0518 \times$ expression of CAPN8 $+0.1323 \times$

185 expression of FILIP1 $+0.0002 \times$ expression of SPP1. According to the signature, risk score was

186 calculated for each patient and each patient was grouped into "high-risk" and "low-risk"

187 according to the median risk score. The distribution of signature risk score (Fig. 9A), survival

188 status and survival time (Fig. 9B) in the model group were presented. The expression of the

189 seven genes in high and low-risk groups was shown in Fig. 9C. Compared with the low-risk

190 patients, the high-risk patients presented worse OS (Fig. 9D). According to the ROC curve on 5-

191 year OS (Fig. 9E), an area under curve (AUC) value of 0.707 (>0.7) indicated that the

192 prognostic prediction value of the seven-gene signature was reliable.

193 Independence assessment of seven-gene mRNA signature

194 Univariate Cox progression analysis indicated that risk score, age, tumor stage and T status

195 were significantly associated with the OS among LUSC patients. Subsequently, multivariate Cox

196 analysis showed that the gene signature had a significantly independent prognostic value with $P$

$197<0.001$ (Table 3). These results revealed that the seven-gene risk signature was an independent

198 prognostic factor for LUSC after adjusting the confounding effects.

\section{Discussion}

200 In this study, a total of 116 up-regulated DEGs and 84 down-regulated DEGs were

201 identified from the GEO database. KEGG enrichment analysis showed that these DEGs were 
202 mainly involved in two pathways: "Cell cycle" and "p53 signaling pathway". Increasing 203 evidences suggest that disordered cell cycle has been a mark of tumors (Hanahan \& Weinberg, 204 2011). In LUSC, cell cycle progression and cell proliferation could be inhibited by CCNB1 205 (Wang et al., 2019), promoted by DDA1 (Cheng et al., 2017), and arrested in the G phase by 206 SART3 (Sherman et al., 2019). Besides, the cell cycle is closely related with the p53 pathway 207 (Joerger and Fershthe, 2016). P53, a multifunctional transcription factor in cancer progression, 208 participates in the regulation of cell cycle, metabolic pathways, and so on (Stegh, 2012; Fridman $209 \&$ Lowe, 2003; Vogelstein et al., 2000). Studies have found that p53 signaling pathway could 210 induce cancer cell apoptosis by targeting at Bax (Jin et al., 2017), p21, and HIF1 $\alpha$ (Yang et al., 211 2018) in LUSC. Besides, p53 plays the role in tumor suppression in LUSC via being regulated 212 by other genes, such as miR-223-3p (Luo et al., 2019), which has not been found in the studies of 213 other cancers. In short, the enriched pathways of these DEGs could interact with each other and 214 participate in the regulation of LUSC progression.

215 In order to predict the function associations between the 200 DEGs, a PPI network was 216 constructed and ten hub genes were identified, including CDK1, BUB1, CCNB2, CCNA2, 217 KIF2C, AURKB, KIF11, MAD2L1, TOP2A, and DLGAP5. Survival analysis revealed that five 218 of hub genes were associated with FP or PPS. Among the five hub genes, the altered CCNA2, 219 DLGAP5, MAD2L1 and KIF2C were associated with tumor grade, and were significantly up220 regulated in LUSC compared with other NSCLC, implicating crucial roles of them in LUSC 221 progression.

CCNA2, also known as CyclinA2, a highly conserved cyclin protein (Ko et al., 2013), has 223 been found significantly over-expressed in various cancers (Gao et al., 2014). Previous

224 225 226 227 228 229 230 231 researches showed that CCNA2 could regulate cell cycle in cancers via controlling the G1/S and G2/M transitions (Arsic et al., 2012). CCNA2 is also associated with epithelial-mesenchymal transition and cancer progression (Bendris et al., 2012). Previous studies have shown that diverse molecules, such as coiled-coil domain containing 6, Zinc finger SWIM-type containing 5, and miR-137, could regulate the proliferation, invasion, and migration of NSCLC cells by changing the expression of CCNA2 (Morra et al., 2015; Xu et al., 2018; Chen et al., 2017). In the present study, CCNA2 was found up-regulated in LUSC tissues compared with normal tissues, and overexpression of CCNA2 was related to worse first-progression survival in LUSC patients. These 
232 results indicated that CCNA2 might be a progression biomarker and prognostic indictor for 233 LUSC.

234 Kinesin family member 11 (KIF11), a kinesin-5-family protein, could affect tumor 235 development by controlling the correct arrangement of the microtubules, which was the key 236 stage in mitosis (Blangy et al., 1995). Previous studies have reported that KIF11 was up237 regulated in lung cancer tissues compared with normal tissues, and associated with poor overall 238 survival (Al-Khafaji et al., 2017; Schneider et al., 2017). Kato et al declared that high-level 239 KIF11 was as an independent prognostic factor in LUSC, and might be promising therapeutic 240 option for advanced lung cancer (Kato et al., 2018). In this study, KIF11 was both identified as 241 one of over-expressed hub genes and related to unfavorable first-progression survival of LUSC 242 patients, which was consistent with previous studies.

243 MAD2 mitotic arrest deficient-like 1 (MAD2L1), an important spindle checkpoint protein, 244 plays important roles in protecting cells from abnormal chromosome segregation ( $Y u, 2006)$. 245 MAD2L1 has been shown high expression levels and might be a biomaker for poor prognosis in 246 various cancers, such as breast cancer (Wang et al., 2015), osteosarcoma (Sun, Li \& Yan, 2015), 247 and so on. MAD2L1 has been identified as a potential therapeutic target gene in NSCLC (Zhou 248 et al., 2015), and could be a promising prognostic biomarker for LUAD (MacDermed et al., 2010) and small cell lung cancer (Liao et al., 2019), however, there was no similar results in LUSC. In this study, over-expression of MAD2L1 and its prognostic value in LUSC were 251 identified.

252 Kinesin family member 2C (KIF2C) has been served as a modulator in bipolar spindle 253 formation, microtubule depolymerization, and chromosome segregation, and it could promote

254

255 256

257 258 259 260 261 the tumor proliferation and metastasis (Gan et al., 2019). In this study, down-regulated KIF2C had a significant value in post-progression survival in LUSC. These suggest that KIF2C might be involved in tumor progression and be a potential prognostic factor in LUSC. Disc large (drosophila) homolog-associated protein 5 (DLGAP5), as an important mitotic spindle protein, participated in cancers development and progression (Liao et al., 2013). DLGAP5 has been found significantly over-expressed in different subtypes of lung cancer, and it could be a promising prognostic biomarker and therapeutic target (Qi et al., 2019), which are consistent with the results of this study. 
262

263

264

265

266

267

268

269

270

271

272

273

274

275

276

277

278

279

280

281

282

283

284

285

286

287

288

289

290

291

292

Proteins encoded by CDK1, BUB1 and AURKB belong to the serine/threonine kinases family, and overexpression of them has been detected involved in various tumors prognosis via regulating tumor cell cycle. CDK1 (Xie et al., 2019) and BUB1 (Piao et al., 2019) have been proved to influence tumor progression by inducing cell cycle dysregulation. AURKB was proved to be a therapeutic target by triggering G1/S arrest in NSCLC (Bertran-Alamillo et al., 2019). CCNB2, encoding the member of the cyclin family, could promote tumor progression by facilitating cell proliferation and maintain normal G2/M transition. CCNB2 and BUB1 might be involved in the cancer stem cells to promote LUSC progression (Qin et al., 2020). TOP2A encoding a DNA topoisomerase, could affect overall survival and clinicopathological features in NSCLC by altering the transcription of DNA (Hou et al., 2017). Much further investigations on the roles of the ten genes in LUSC are needed. In this report, functional analysis and their roles in other tumors might provide valuable clues to investigate their roles in LUSC progression.

In this study, none of the ten hub gene was found associated with OS in LUSC by K-M plotter analysis and cox progression analysis using TCGA database. Hence, cox progression analysis was performed for all the DEGs. Compared to a single gene marker to predict patient survival, a gene signature will provide a stable and effective prediction effect. Therefore, a novel seven-gene signature (including FLRT3, PPP2R2C, MMP3, MMP12, CAPN8, FILIP1, and SPP1) was established for LUSC prognostic prediction. According to the gene signature, each LUSC patient with a risk score was classified as high-risk or low-risk. The risk scores of highrisk patients were much higher than that of low-risk patients, which proved that the risk score was significant. Results showed that high-risk patients presented significantly worse OS than low-risk patients. ROC demonstrated that the seven-gene signature was efficient and sensitive in the survival prediction in LUSC. In addition, clinical pathological features (including age, gender, grade, and T/M/N state) are known prognostic factors in LUSC. In order to avoid the interference effect of these known prognostic factors, multivariate Cox regression analysis was carried out, and proved that the signature was an independent prognostic factor in LUSC. All these results suggested that the signature could be an efficient and independent indicator for LUSC prognosis.

Bluemn et al. demonstrated the loss of PPP2R2C is associated with cancer recurrence and a poor survival in prostate cancer (Bluemn et al., 2013). Nevertheless, the role of PPP2R2C in LUSC remains unclear. Previous studies have proved MMP3 (matrix metalloproteinase 3) played 
293 crucial roles in invasion and metastasis in many cancers (Ma et al., 2019). MMP3 is associated

294 with poor survival in various cancers, and its polymorphism might increase the risk of lung 295 cancer (Hu et al., 2013). Up-regulated MMP12 has been found associated with the pathological 296 stage and tumor metastasis in lung cancer (Lv et al., 2015). CAPN8 has been proved up297 regulated in lung cancer and could be a prognostic biomarker (Zhang et al., 2015). FILIP1L 298 (FILamin A Interacting Protein 1-Like) is involved in cell proliferation and migration by 299 inhibiting the WNT signaling pathway (Kwon et al., 2014). Up-regulated SPP1 (secreted 300 phosphoprotein 1) could promote cell proliferation, migration, and invasion by Integrin $301 \beta 1 / F A K / A K T$ pathway (Zeng et al.,2018), and could be a prognostic indicator in many tumors 302 (Choe et al.,2018). Inhibition of SPP1 could enhance the invasion and might be a promising 303 target for NSCLC therapy (Wang et al., 2019). To our knowledge, the seven-gene signature for 304 305 prognostic prediction in LUSC has not been reported previously, and has been demonstrated as an independent and useful prognostic signature in LUSC.

In addition, in the study, many more genes are specific to one database but not the others. This might be caused by the heterogeneity between different datasets. Various factors might lead to the heterogeneity, such as experimental methods and conditions, detection platform and system, sample sources, reagent batches, and so on. Some aspects in the present study have been considered to reduce the influences. Firstly, all of the samples in this study are from normal lung tissues and LUSC tissues, and not from adjacent tissues, which ensures the consistency of the experimental grouping. Secondly, all the data from the GEO database have been normalized, which could reduce variation between groups and make them comparable. Lastly, the DEGs were identified from each dataset, and then the common part of them were selected as the final DEGs, which could reduce the influences resulted from the heterogeneity of the different datasets. These aspects could make the analysis results more effective and objective.

\section{Conclusions}

318 In summary, this study identified 200 DEGs between LUSC and normal tissues by three 319 GEO datasets. Ten up-regulated hub genes were validated in TCGA database and were 320 associated with cell cycle and p53 signaling pathway. CCNA2, DLGAP5, MAD2L1, and KIF2C were significantly up-regulated compared to other subtypes, and associated with tumor stage in LUSC, suggesting that they might tightly be involved in progression and prognosis of LUSC. In 
323 addition, a novel seven-gene signature was established to predict overall survival in LUSC,

324 which may help to provide clues in LUSC prognosis.

325

326

327

328

329

330

331

332

333

334

335

336

337

338

339

340

341

342

343

344

345

346

347

348

349

350

351

352

\section{Acknowledgements}

The authors would like to thank Dr. Shan Shan Li affiliated with CapitalBio Technology

Company (Beijing), for her technical assistance.

\section{References}

Al-Khafaji ASK, Marcus MW, Davies MPA, Risk JM, Shaw RJ, Field JK, Liloglou T. 2017. AURKA mRNA expression is an independent predictor of poor prognosis in patients with non-small cell lung cancer. Oncol Lett 13:4463-4468 DOI 10.3892/ol.2017.6012.

Arsic N, Bendris N, Peter M, Begon-Pescia C, Rebouissou C, Gadea G, Bouquier N, Bibeau F, Lemmers B, Blanchard JM. 2012. A novel function for Cyclin A2: control of cell invasion via RhoA signaling. J Cell Biol 196:147-162 DOI 10.1083/jcb.201102085.

Bendris N, Arsic N, Lemmers B, Blanchard JM. 2012. Cyclin A2, Rho GTPases and EMT. Small GTPases 3:225-228 DOI 10.4161/sgtp.20791.

Bertran-Alamillo J, Cattan V, Schoumacher M, Codony-Servat J, Giménez-Capitán A, Cantero F, Burbridge M, Rodríguez S, Teixidó C, Roman R, Castellví J, García-Román S, Codony-Servat C, Viteri S, Cardona AF, Karachaliou N, Rosell R, Molina-Vila MA. 2019. AURKB as a target in non-small cell lung cancer with acquired resistance to anti-EGFR therapy. Nat Commun 10(1):1812. DOI 10.1038/s41467-019-09734-5

Blangy A, Lane HA, d'Herin P, Harper M, Kress M, Nigg EA. 1995. Phosphorylation by p34cdc2 regulates spindle association of human Eg5, a kinesin-related motor essential for bipolar spindle formation in vivo. Cell 83:1159-1169 DOI 10.1016/0092-8674(95)90142-6.

Bluemn EG, Spencer ES, Mecham B, Gordon RR, Coleman I, Lewinshtein D, Mostaghel E, Zhang XT., Annis J, Grandori C, Porter C, Nelson PS. 2013. PPP2R2C loss promotes castration-resistance and is associated with increased prostate cancer-specific mortality. Mol Cancer Res 11(6): 568-78. DOI 10.1158/1541-7786.MCR-12-0710 
353 Bray F, Ferlay J, Soerjomataram I, Siegel RL, Torre LA, and Jemal A. 2018. Global cancer

354 statistics 2018: GLOBOCAN estimates of incidence and mortality worldwide for 36 cancers in 355185 countries. CA Cancer J Clin 68:394-424 DOI 10.3322/caac.21492.

356 Calvayrac O, Pradines A, Pons E, Mazieres J, Guibert N. 2017. Molecular biomarkers for lung 357 adenocarcinoma. Eur Respir J 49 DOI 10.1183/13993003.01734-2016.

358 Chen R, Zhang Y, Zhang C, Wu H, Yang S. 2017. miR-137 inhibits the proliferation of human 359 non-small cell lung cancer cells by targeting SRC3. Oncol Lett 13:3905-3911 DOI $360 \quad 10.3892 / \mathrm{ol} .2017 .5904$.

361 Chen Z, Fillmore CM, Hammerman PS, Kim CF, Wong KK. 2014. Non-small-cell lung cancers: 362 a heterogeneous set of diseases. Nat Rev Cancer 14:535-546.

363 Choe EK, Yi JW, Chai YJ, Park KJ. 2018. Upregulation of the adipokine genes ADIPOR1 and 364 SPP1 is related to poor survival outcomes in colorectal cancer. J Surg Oncol 117(8): 1833365 1840. DOI 10.1002/jso.25078.

366 Fridman JS, Lowe SW. 2003. Control of apoptosis by p53. Oncogene 22:9030-9040 DOI $367 \quad 10.1038 / \mathrm{nrc} 3775$.

368 Gan H, Lin L, Hu N, Yang Y, Gao Y, Pei Y, Chen K, Sun B. 2019. KIF2C exerts an oncogenic 369 role in nonsmall cell lung cancer and is negatively regulated by miR-325-3p. Cell Biochem Funct 37:424-431 DOI 10.1002/cbf.3420.

Gao T, Han Y, Yu L, Ao S, Li Z, Ji J. 2014. CCNA2 is a prognostic biomarker for ER+ breast cancer and tamoxifen resistance. PLoS One 9:e91771 DOI 10.1371/journal.pone.0091771.

Hanahan D, and Weinberg RA. 2011. Hallmarks of cancer: the next generation. CELL 144:646674 DOI 10.1016/j.cell.2011.02.013.

Hirsch FR, Scagliotti GV, Mulshine JL, Kwon R, Curran WJ, Jr., Wu YL, Paz-Ares L. 2017. Lung cancer: current therapies and new targeted treatments. Lancet 389:299-311 DOI 10.1016/S0140-6736(16)30958-8.

Hou GX, Liu PP, Yang J., Wen SJ. 2017. Mining expression and prognosis of topoisomerase isoforms in non-small-cell lung cancer by using Oncomine and Kaplan-Meier plotter. PLoS ONE 12(3): e0174515. DOI 10.1371/journal.pone.0174515

Hou Z, Xu C, Xie H, Xu H, Zhan P, Yu L, Fang X. 2014. Long noncoding RNAs expression patterns associated with chemo response to cisplatin based chemotherapy in lung squamous cell carcinoma patients. PLoS One 9:e108133 DOI 10.1371/journal.pone.0108133. 
384 Hu CL, Wang JM, Xu YZ, Li XC, Chen HL, Bunjhoo H., Xiong WN, Xu YJ, Zhao JP. 2013. 385 Current evidence on the relationship between five polymorphisms in the matrix

386

387

388

389

390

391

392

393

394

395

396

397

398

399

400

401

402

403

404

405

406

407

408

409

410

411

412

413

414

metalloproteinases (MMP) gene and lung cancer risk: a meta-analysis. Gene 517(1):65-71

DOI 10.1016/j.gene.2012.12.085

Hwang JA, Song JS, Yu DY, Kim HR, Park HJ, Park YS, Kim WS, Choi CM. 2015.

Peroxiredoxin 4 as an independent prognostic marker for survival in patients with early-stage lung squamous cell carcinoma. Int J Clin Exp Pathol 8:6627-6635.

Jin C, Zhang G, Zhang Y, Hua P, Song G, Sun M, Li X, Tong T, Li B, Zhang X. 2017.

Isoalantolactone induces intrinsic apoptosis through p53 signaling pathway in human lung squamous carcinoma cells. PLoS One 12(8):e0181731 DOI 10.1371/journal.pone.0181731.

Joerger AC, and Fersht AR. 2016. The p53 Pathway: Origins, Inactivation in Cancer, and Emerging Therapeutic Approaches. Annu. Rev. Biochem 85: 375-404 DOI 10.1146/annurevbiochem-060815-014710

Kato T, Lee D, Huang H, Cruz W, Ujiie H, Fujino K, Wada H, Patel P, Hu HP, Hirohashi K, Nakajima T, Sato M, Kaji M, Kaga K, Matsui Y, Chen J, Zheng G, Yasufuku K. 2018. Personalized siRNA-Nanoparticle Systemic Therapy using Metastatic Lymph Node Specimens Obtained with EBUS-TBNA in Lung Cancer. Mol Cancer Res 16:47-57 DOI 10.1158/1541-7786.MCR-16-0341.

Ko E, Kim Y, Cho EY, Han J, Shim YM, Park J, Kim DH. 2013. Synergistic effect of Bcl-2 and cyclin A2 on adverse recurrence-free survival in stage I non-small cell lung cancer. Ann Surg Oncol 20:1005-1012 DOI 10.1245/s10434-012-2727-2.

Kuo CN, Liao YM, Kuo LN, Tsai HJ, Chang WC, Yen Y. 2019. Cancers in Taiwan: Practical insight from epidemiology, treatments, biomarkers, and cost. J Formos Med Assoc S09296646(19):30018-X DOI 10.1016/j.jfma.2019.08.023.

Kwon M, Lee SJ, Wang Y, Rybak Y, Luna A, Reddy S, Adem A, Beaty BT, Condeelis JS, Libutti SK. 2014. Filamin A interacting protein 1-like inhibits WNT signaling and MMP expression to suppress cancer cell invasion and metastasis. Int J Cancer 135(1):48-60 DOI 10.1002/ijc.28662.

Li S, Sun X, Miao S, Liu J, Jiao W. 2017. Differential protein-coding gene and long noncoding RNA expression in smoking-related lung squamous cell carcinoma. Thorac Cancer 8:672-681 DOI 10.1111/1759-7714.12510.

Peer) reviewing PDF | (2019:09:41593:1:1:CHECK 8 Mar 2020) 
415 Liao W, Liu W, Yuan Q, Liu X, Ou Y, He S, Yuan S, Qin L, Chen Q, Nong K, Mei M, Huang J. 416 2013. Silencing of DLGAP5 by siRNA significantly inhibits the proliferation and invasion of 417 hepatocellular carcinoma cells. PLoS One 8:e80789 DOI 10.1371/journal.pone.0080789. 418 Liao Y, Yin G, Wang X, Zhong P, Fan X, Huang C. 2019. Identification of candidate genes 419 associated with the pathogenesis of small cell lung cancer via integrated bioinformatics 420 analysis. Oncol Lett 18:3723-3733 DOI 10.3892/ol.2019.10685.

421 Luo P, Wang Q, Ye YY., Zhang J, Lu DP, Cheng LQ, Zhou HC, Xie MG, Wang BL. 2019. 422 MiR-223-3p functions as a tumor suppressor in lung squamous cell carcinoma by miR-223423 3p-mutant p53 regulatory feedback loop. J Exp Clin Cancer Res 38(1):74 DOI 424 10.1186/s13046-019-1079-1.

425 Lv FZ, Wang JL, Wu Y, Chen HF, Shen XY. 2015. Knockdown of MMP12 inhibits the growth 426 and invasion of lung adenocarcinoma cells. Int J Immunopathol Pharmacol 28(1): 77-84 DOI $427 \quad 10.1177 / 0394632015572557$.

428 Ma YH, Cang SD, Li GQ, Su Y, Zhang HF, Wang LM., Yang JP, Shi XY, Qin GJ, Yuan HJ. 429 2019. Integrated analysis of transcriptome data revealed MMP3 and MMP13 as critical genes 430 in anaplastic thyroid cancer progression. J Cell Physiol 234(12): 22260-22271 DOI $431 \quad 10.1002 /$ jcp.28793.

432 MacDermed DM, Khodarev NN, Pitroda SP, Edwards DC, Pelizzari CA, Huang L, Kufe DW, 433 Weichselbaum RR. 2010. MUC1-associated proliferation signature predicts outcomes in lung 434 adenocarcinoma patients. BMC Med Genomics 3:16 DOI 10.1186/1755-8794-3-16.

435 Morra F, Luise C, Visconti R, Staibano S, Merolla F, Ilardi G, Guggino G, Paladino S, Sarnataro 436 D, Franco R, Monaco R, Zitomarino F, Pacelli R, Monaco G, Rocco G, Cerrato A, 437 Linardopoulos S, Muller MT, Celetti A. 2015. New therapeutic perspectives in CCDC6 438 deficient lung cancer cells. Int J Cancer 136:2146-2157 DOI 10.1002/ijc.29263.

439 Piao JJ, Zhu LH, Sun J, Li N, Dong B, Yang Y, Chen LY. 2019. High expression of CDK1 and 440 BUB1 predicts poor prognosis of pancreatic ductal adenocarcinoma. Gene 701:15-22 DOI $441 \quad$ 10.1016/j.gene.2019.02.081.

442 Qi L, Gao C, Feng F, Zhang T, Yao Y, Wang X, Liu C, Li J, Li J, Sun C. 2019. MicroRNAs 443 associated with lung squamous cell carcinoma: New prognostic biomarkers and therapeutic 444 targets. J Cell Biochem 120:18956-18966 DOI 10.1002/jcb.29216. 
445 Qin SB, Long X, Zhao Q, Zhao WX. 2020. Co-Expression Network Analysis Identified Genes 446 Associated with Cancer Stem Cell Characteristics in Lung Squamous Cell Carcinoma. Cancer 447 Invest. 38(1):13-22 DOI 10.1080/07357907.2019.1697281.

448 Schneider MA, Christopoulos P, Muley T, Warth A, Klingmueller U, Thomas M, Herth FJ, 449 Dienemann H, Mueller NS, Theis F, Meister M. 2017. AURKA, DLGAP5, TPX2, KIF11 and 450 CKAP5: Five specific mitosis-associated genes correlate with poor prognosis for non-small 451 cell lung cancer patients. Int J Oncol 50:365-372 DOI 10.3892/ijo.2017.3834.

452 Stegh AH. 2012. Targeting the p53 signaling pathway in cancer therapy - the promises,

453 challenges and perils. Expert Opin Ther Targets 16(1): 67-83 DOI $454 \quad 10.1517 / 14728222.2011 .643299$.

455 Sun KX, Zheng RS, Zeng HM, Zhang SW, Zou XN, Gu XY, Xia CF, Yang ZX, Li H, Chen 456 WQ, He J. 2018. The incidence and mortality of lung cancer in China, 2014. Zhonghua Zhong 457 Liu Za Zhi 40:805-811 DOI 10.3760/cma.j.issn.0253-3766.2018.11.002.

458 Sun L, Li J, Yan B. 2015. Gene expression profiling analysis of osteosarcoma cell lines. Mol 459 Med Rep 12:4266-4272 DOI 10.3892/mmr.2015.3958.

460 Vogelstein B, Lane D, Levine AJ, Levine AJ. 2000. Surfing the p53 network. Nature (London) 461 408:307-310. DOI 10.1038/35042675

462 Wang X, Zhang F, Yang X, Xue M, Li X, Gao Y, Liu L. 2019. Secreted Phosphoprotein 1 (SPP1) 463 Contributes to Second-Generation EGFR Tyrosine Kinase Inhibitor Resistance in Non-Small 464 Cell Lung Cancer. Oncol Res 27(8):871-877 DOI 10.3727/096504018X15426271404407. 465 Wang X, Zhang F, Yang X, Xue M, Li X, Gao Y, Liu L. 2019. Secreted Phosphoprotein 1 (SPP1) 466 Contributes to Second-Generation EGFR Tyrosine Kinase Inhibitor Resistance in Non-Small 467 Cell Lung Cancer. Oncol Res 27(8): 871-877 DOI 10.3727/096504018X15426271404407. 468 Wang Z, Katsaros D, Shen Y, Fu Y, Canuto EM, Benedetto C, Lu L, Chu WM, Risch HA, Yu H. 469 470 471 472 473 in cell cycle progression and tumor resistance. Cancer Lett 443: 56-66 DOI $474 \quad 10.1016 / j . c a n l e t .2018 .11 .019$. 
475 Xu J, Shu Y, Xu T, Zhu W, Qiu T, Li J, Zhang M, Xu J, Guo R, Lu K, Zhu L, Yin Y, Gu Y, Liu

476 L, Liu P, Wang R. 2018. Microarray expression profiling and bioinformatics analysis of

477 circular RNA expression in lung squamous cell carcinoma. Am J Transl Res 10:771-783.

478 Xu J, Shu Y, Xu T, Zhu W, Qiu T, Li J, Zhang M, Xu J, Guo R, Lu K, Zhu L, Yin Y, Gu Y, Liu

479 L, Liu P, Wang R. 2018. Microarray expression profiling and bioinformatics analysis of

480 circular RNA expression in lung squamous cell carcinoma. Am J Transl Res 10:771-783.

481 Yang D, Cheng DM, Tu Q, Yang HH, Sun B, Yan LZ, Dai HJ, Luo JR, Mao BY, Cao Y, Yu XP,

482 Jiang H, Zhao XD. 2018. HUWE1 controls the development of non-small cell lung cancer

483 through down-regulation of p53. Theranostics 8(13):3517-3529 DOI 10.7150/thno.24401.

484 Yeo MK, Choi SY, Seong IO, Suh KS, Kim JM, Kim KH. 2017. Association of PD-L1

485 expression and PD-L1 gene polymorphism with poor prognosis in lung adenocarcinoma and

486 squamous cell carcinoma. Hum Pathol 68:103-111 DOI 10.1016/j.humpath.2017.08.016.

$487 \mathrm{Yu} \mathrm{H.} \mathrm{2006.} \mathrm{Structural} \mathrm{activation} \mathrm{of} \mathrm{Mad2} \mathrm{in} \mathrm{the} \mathrm{mitotic} \mathrm{spindle} \mathrm{checkpoint:} \mathrm{the} \mathrm{two-state} \mathrm{Mad2}$

488 model versus the Mad2 template model. J Cell Biol 173:153-157 DOI 10.1083/jcb.200601172.

489 Zeng B, Zhou M, Wu H, Xiong Z. 2018. SPP1 promotes ovarian cancer progression via Integrin

$490 \quad \beta 1 / F A K / A K T$ signaling pathway. Onco Targets Ther 11:1333-1343 DOI

$491 \quad$ 10.2147/OTT.S154215. eCollection 2018.

492 Zhang Y, Wang H, Wang J, Bao L, Wang L, Huo J, Wang X. 2015. Global analysis of

493 chromosome 1 genes among patients with lung adenocarcinoma, squamous carcinoma, large-

494 cell carcinoma, small-cell carcinoma, or non-cancer. Cancer Metastasis Rev 34(2):249-64 DOI

$49510.1007 / \mathrm{s} 10555-015-9558-0$.

496 Zhou W, Yin M, Cui H, Wang N, Zhao LL, Yuan LZ, Yang XP, Ding XM, Men FZ, Ma X, Na

497 JR. 2015. Identification of potential therapeutic target genes and mechanisms in non-small-cell

498 lung carcinoma in non-smoking women based on bioinformatics analysis. Eur Rev Med

499 Pharmacol Sci 19:3375-3384.

500 


\section{Table 1 (on next page)}

Significantly enriched GO terms and KEGG pathways of DEGs. 


\begin{tabular}{|c|c|c|c|c|}
\hline $\begin{array}{l}\text { Categor } \\
\mathbf{y}\end{array}$ & Term & Description & Count & FDR \\
\hline $\mathrm{BP}$ & GO:0007067 & mitotic nuclear division & 27 & $8.64 \mathrm{E}-15$ \\
\hline $\mathrm{BP}$ & GO:0051301 & cell division & 28 & $4.98 \mathrm{E}-12$ \\
\hline $\mathrm{BP}$ & GO:0007062 & sister chromatid cohesion & 15 & $1.60 \mathrm{E}-08$ \\
\hline $\mathrm{BP}$ & GO:0007059 & chromosome segregation & 12 & $4.09 \mathrm{E}-07$ \\
\hline $\mathrm{BP}$ & GO:0000082 & G1/S transition of mitotic cell cycle & 12 & $3.40 \mathrm{E}-05$ \\
\hline $\mathrm{BP}$ & GO:0006260 & DNA replication & 11 & 0.01728675 \\
\hline $\mathrm{BP}$ & GO:0008283 & cell proliferation & 16 & 0.029764593 \\
\hline $\mathrm{BP}$ & GO:0000086 & $\mathrm{G} 2 / \mathrm{M}$ transition of mitotic cell cycle & 10 & 0.041628856 \\
\hline $\mathrm{CC}$ & GO:0000777 & condensed chromosome kinetochore & 14 & $9.87 \mathrm{E}-09$ \\
\hline $\mathrm{CC}$ & GO:0000775 & chromosome, centromeric region & 11 & $5.29 \mathrm{E}-07$ \\
\hline $\mathrm{CC}$ & GO:0030496 & midbody & 14 & $1.54 \mathrm{E}-06$ \\
\hline $\mathrm{CC}$ & GO:0000776 & kinetochore & 10 & $2.51 \mathrm{E}-04$ \\
\hline $\mathrm{CC}$ & GO:0005819 & spindle & 11 & 8.59E-04 \\
\hline $\mathrm{CC}$ & GO:0005578 & proteinaceous extracellular matrix & 15 & 0.00144238 \\
\hline $\mathrm{CC}$ & GO:0005654 & nucleoplasm & 55 & 0.006300472 \\
\hline $\mathrm{CC}$ & GO:0005829 & cytosol & 61 & 0.01409558 \\
\hline $\mathrm{MF}$ & GO:0003777 & microtubule motor activity & 10 & $2.36 \mathrm{E}-04$ \\
\hline KEGG & hsa04110 & Cell cycle & 12 & $1.59 \mathrm{E}-05$ \\
\hline KEGG & hsa04115 & p53 signaling pathway & 7 & 0.037570687 \\
\hline
\end{tabular}


2 Abbreviations: BP, biological process; CC, cellular component; MF, molecular function; DEG, differentially

3 expressed gene; GO, Gene Ontology; KEGG, Kyoto Encyclopedia of Genes and Genomes; FDR, adjust $P$

4 value

5

6 


\section{Table 2 (on next page)}

Prognostic values for the seven-genes prognostic signature in 488 LUSC patients. 
1

2

Table 2 Prognostic values of the seven-genes prognostic signature in 488 LUSC patients

\begin{tabular}{lccccc}
\hline \multicolumn{1}{c}{ id } & coef & HR & HR.95L & HR.95H & $\boldsymbol{P}$ value \\
\hline FLRT3 & 0.034099635 & 1.034687693 & 1.004943783 & 1.065311951 & 0.021943406 \\
PPP2R2C & 0.033850795 & 1.034430253 & 1.000444568 & 1.069570452 & 0.047028752 \\
MMP3 & 0.005741905 & 1.005758421 & 1.000626781 & 1.010916378 & 0.027803799 \\
MMP12 & 0.003057819 & 1.003062498 & 1.000893676 & 1.00523602 & 0.005626057 \\
CAPN8 & 0.051756734 & 1.053119523 & 1.013625297 & 1.094152576 & 0.007956628 \\
FILIP1 & 0.132257889 & 1.141402636 & 1.038057415 & 1.255036532 & 0.006308144 \\
SPP1 & 0.0002455 & 1.00024553 & 1.000013897 & 1.000477217 & 0.037749151 \\
\hline
\end{tabular}


Table 3 (on next page)

Univariate and multivariate Cox regression analysis of OS in LUSC. 
1

Table 3 Univariate and multivariate Cox regression analysis of OS in LUSC

\begin{tabular}{lllllll}
\hline Clinical feature & \multicolumn{3}{c}{ Univariate analysis } & \multicolumn{3}{l}{ Multivariate analysis } \\
\cline { 2 - 7 } & HR & $\mathbf{9 5 \% C I}$ & $\boldsymbol{P}$-value & HR & $\mathbf{9 5 \% C I}$ & $\boldsymbol{P}$-value \\
\hline Risk score(high/low) & 1.655 & $(1.431-1.913)$ & $<0.001$ & 1.642 & $(1.412-1.412)$ & $<0.001$ \\
age(year) & 1.024 & $(1.004-1.045)$ & 0.02 & 1.028 & $(1.006-1.049)$ & 0.011 \\
gender(male/female) & 1.263 & $(0.863-1.849)$ & 0.229 & 1.289 & $(0.878-1.890)$ & 0.195 \\
stage(IV, III/II, I) & 1.261 & $(1.044-1.524)$ & 0.016 & 0.982 & $(0.636-1.518)$ & 0.936 \\
T(T4, T3/T2, T1) & 1.311 & $(1.059-1.623)$ & 0.013 & 1.272 & $(0.925-1.749)$ & 0.138 \\
M(M1/M0) & 1.829 & $(0.581-5.761)$ & 0.302 & 1.816 & $(0.455-7.253)$ & 0.399 \\
N(N3, N2, N1/N0) & 1.159 & $(0.93-1.442)$ & 0.189 & 1.182 & $(0.802-1.742)$ & 0.398 \\
\hline
\end{tabular}

2 


\section{Figure 1}

Volcano plots $(A-C)$ and Venn diagrams (D-E).

(A-C): The DEGs of GSE19188, GSE33479, and GSE33532. The orange dots represent DEGs filtered based on the cutoff criteria of adjusted $P$-value $<0.05$ and $\mid \log 2$ (fold change) $\mid \geq 2$. while the blue dots represent genes that are not satisfied the cutoff criteria. The genes with $\log 2$ (fold change) $\geq 2$ were up-regulated and the genes with log2 (fold change) $\leq-2$ were down-regulated. (D-E): The common up-regulated and down-regulated DEGs in the three datasets.

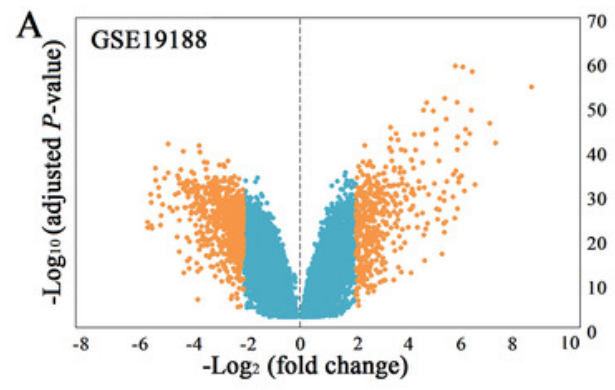

D
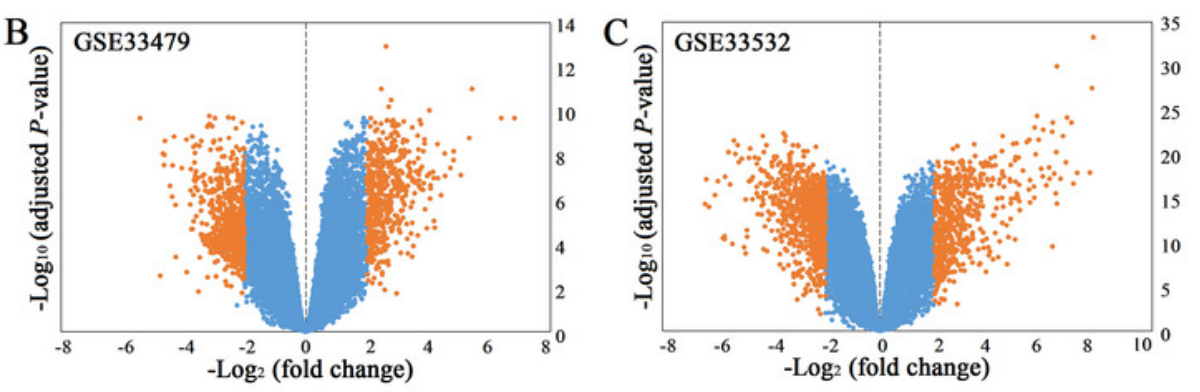

$\mathrm{E}$
Down-regulated

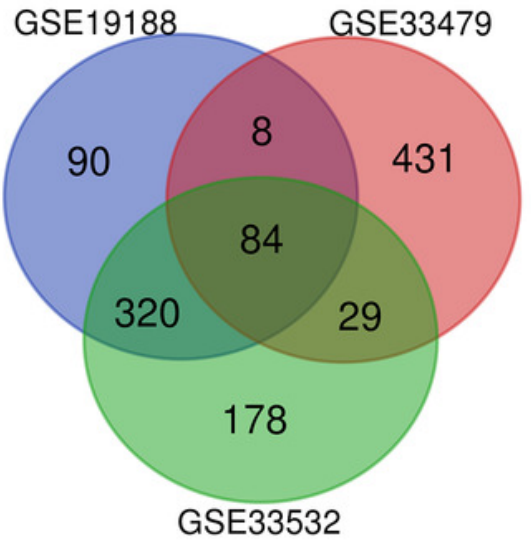




\section{Figure 2}

Enriched GO terms and KEGG pathways of the DEGs.

MF: molecular function; CC: cellular component; BP: biological process. Adjusted $P$ value $($ FDR $)<0.05$ was considered significantly.

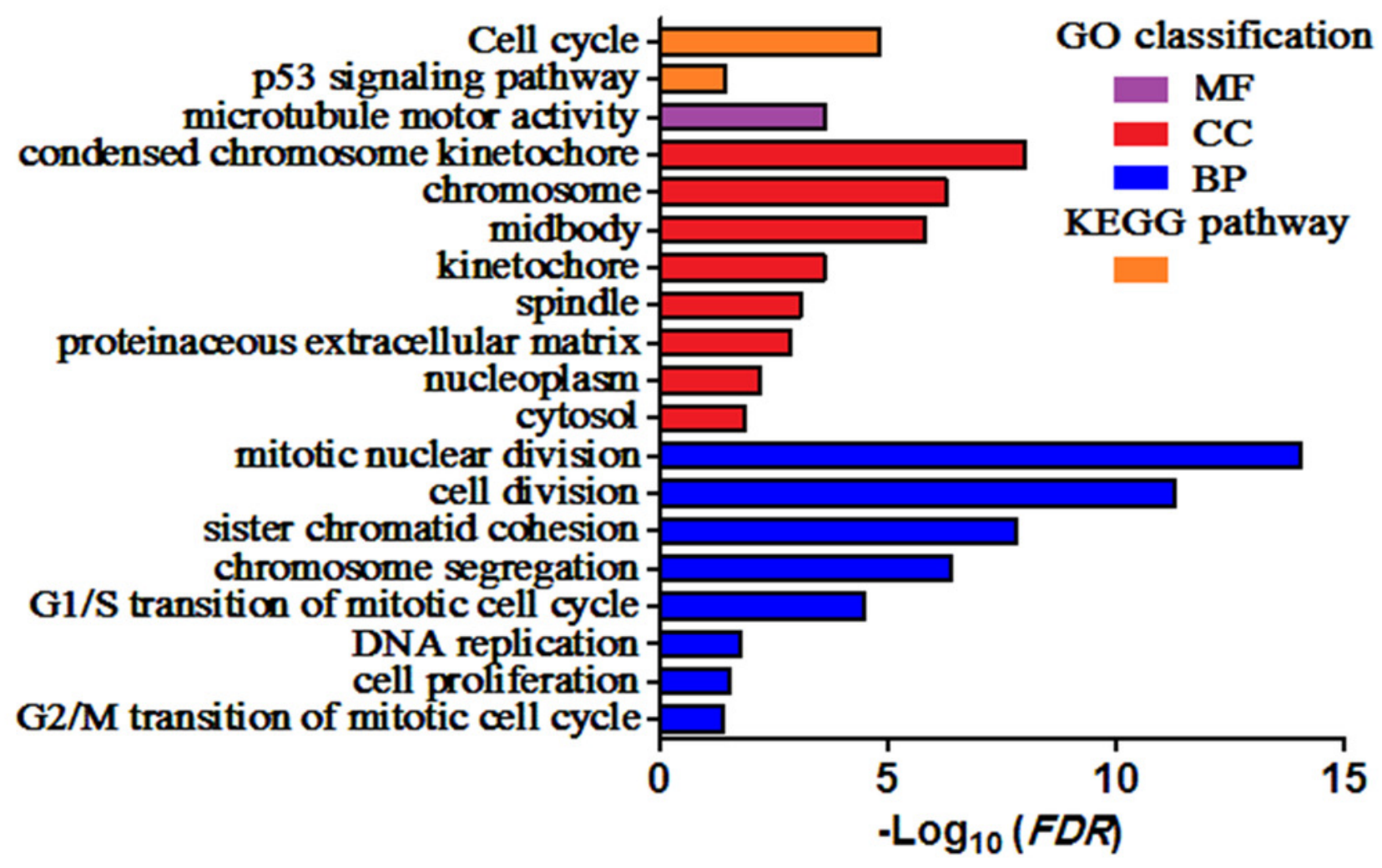


Figure 3

Protein-protein interaction network of DEGs.

The red nodes represent the up-regulated DEGs. The blue nodes represent the downregulated DEGs.

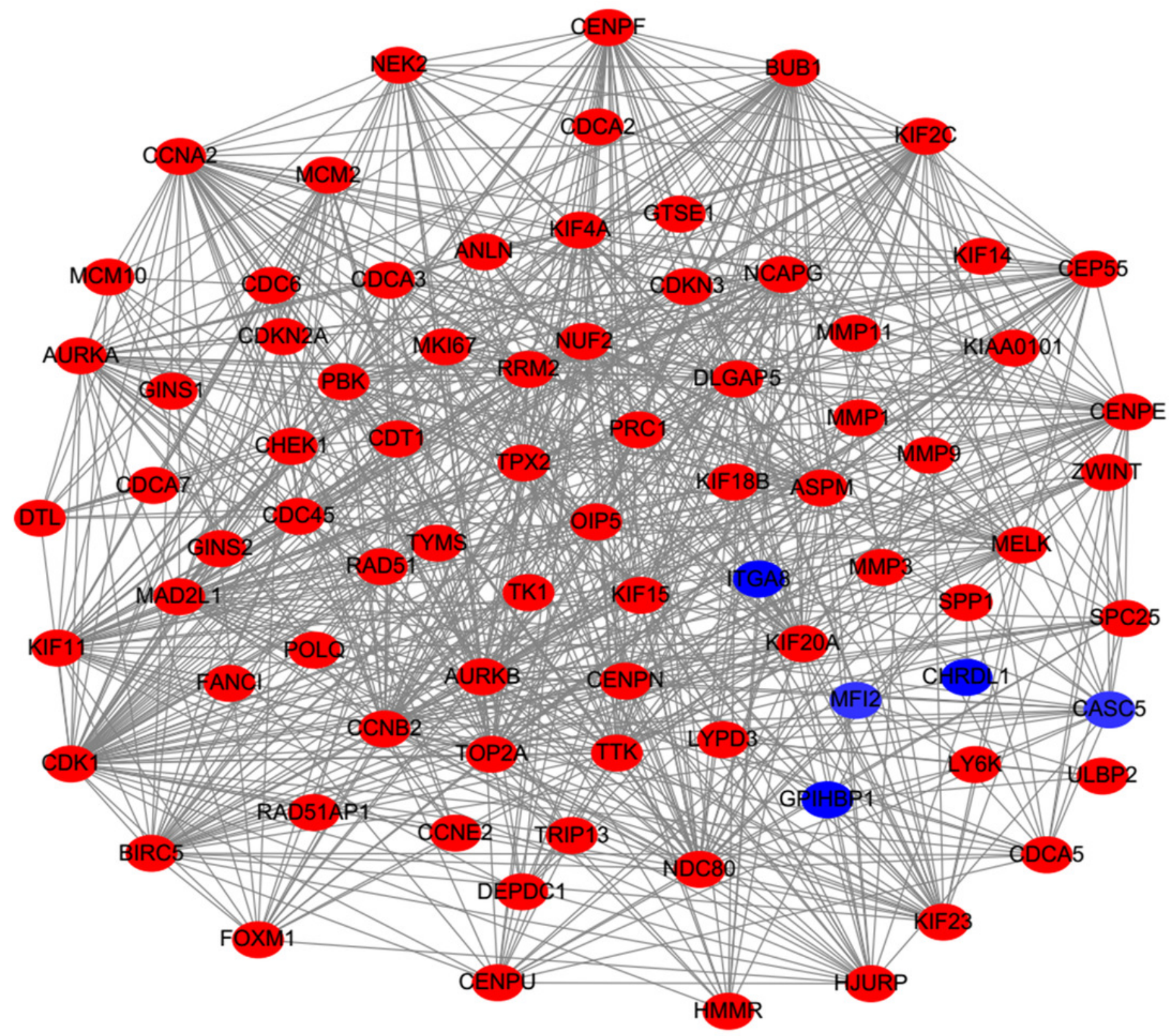




\section{Figure 4}

A PPI network for the hub genes identified with the Cytoscape.

The top ten hub genes were ranked by the degree, and advanced ranking is represented by a redder color.

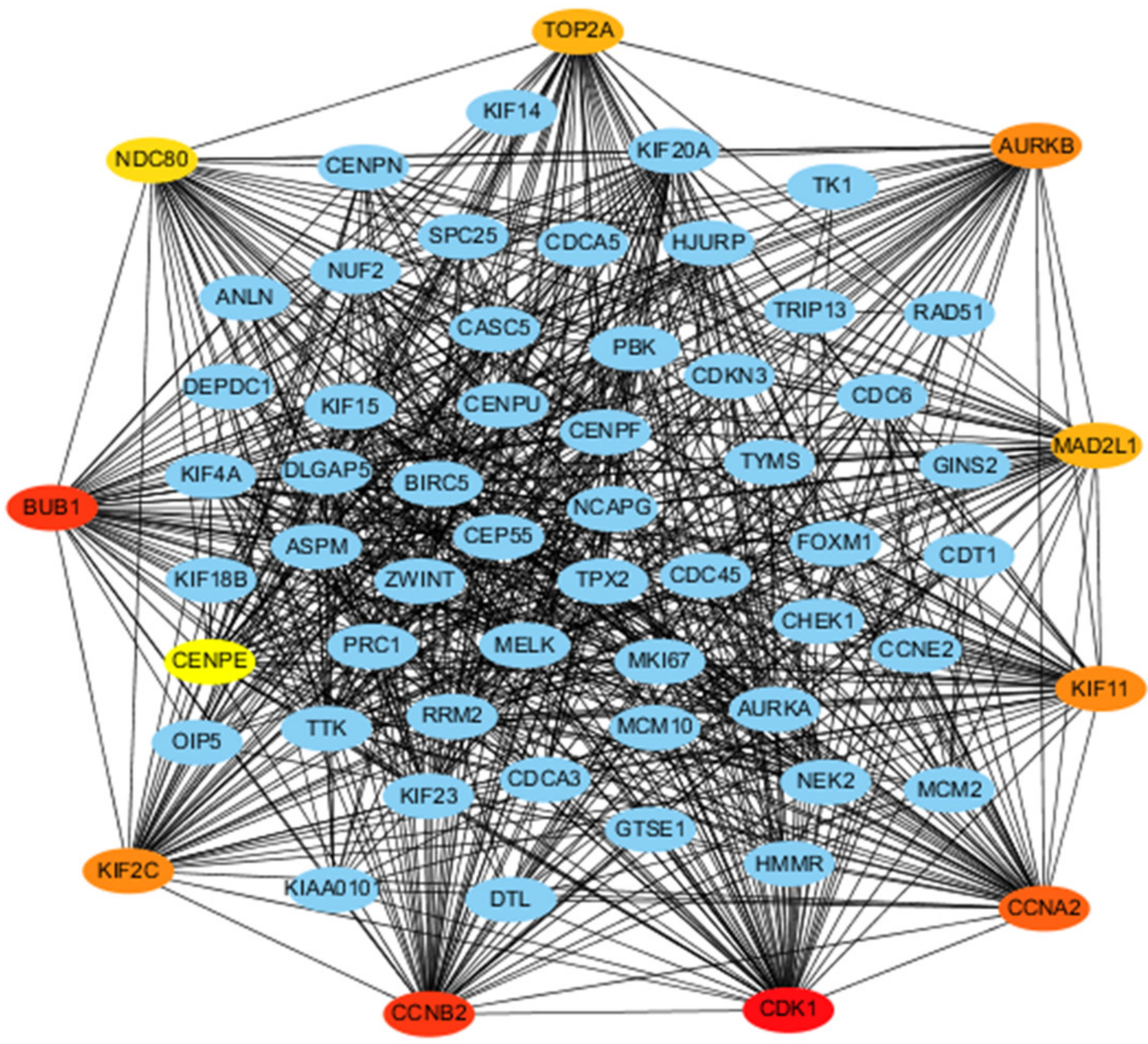


Figure 5

The expression levels of 10 hub genes in the TCGA database.

The expression of the ten hub genes in 502 LUSC tissues and 49 normal tissues from TCGA database. Gene expression values are $\log _{2}$-transformed.

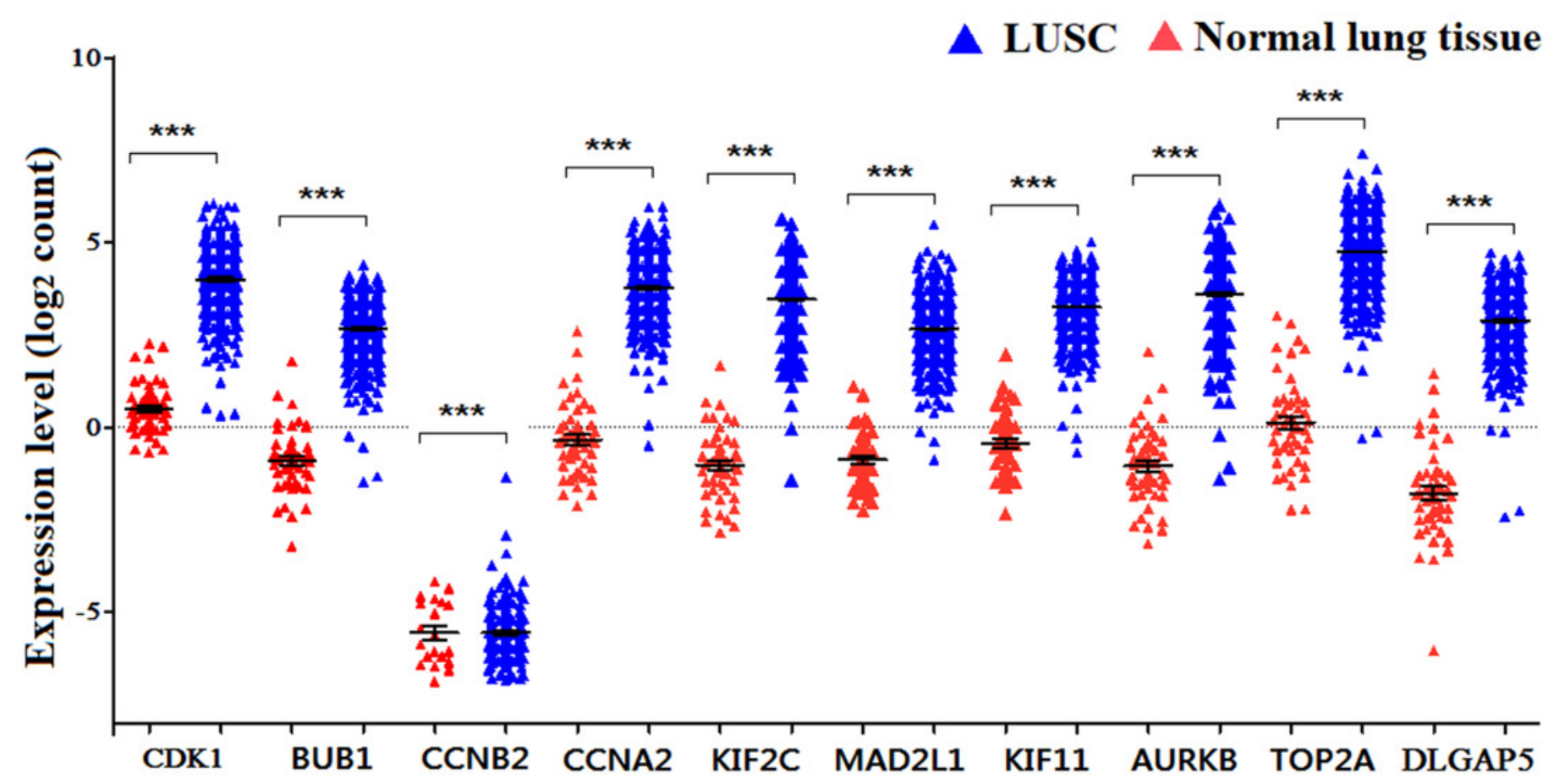


Figure 6

The expression levels of 10 hub genes between different subtypes.

The expression of the ten hub genes in LUSC was significantly different from that in other subtypes, which was analyzed using Oncomine database. Gene expression values are $\log _{2}$ transformed.
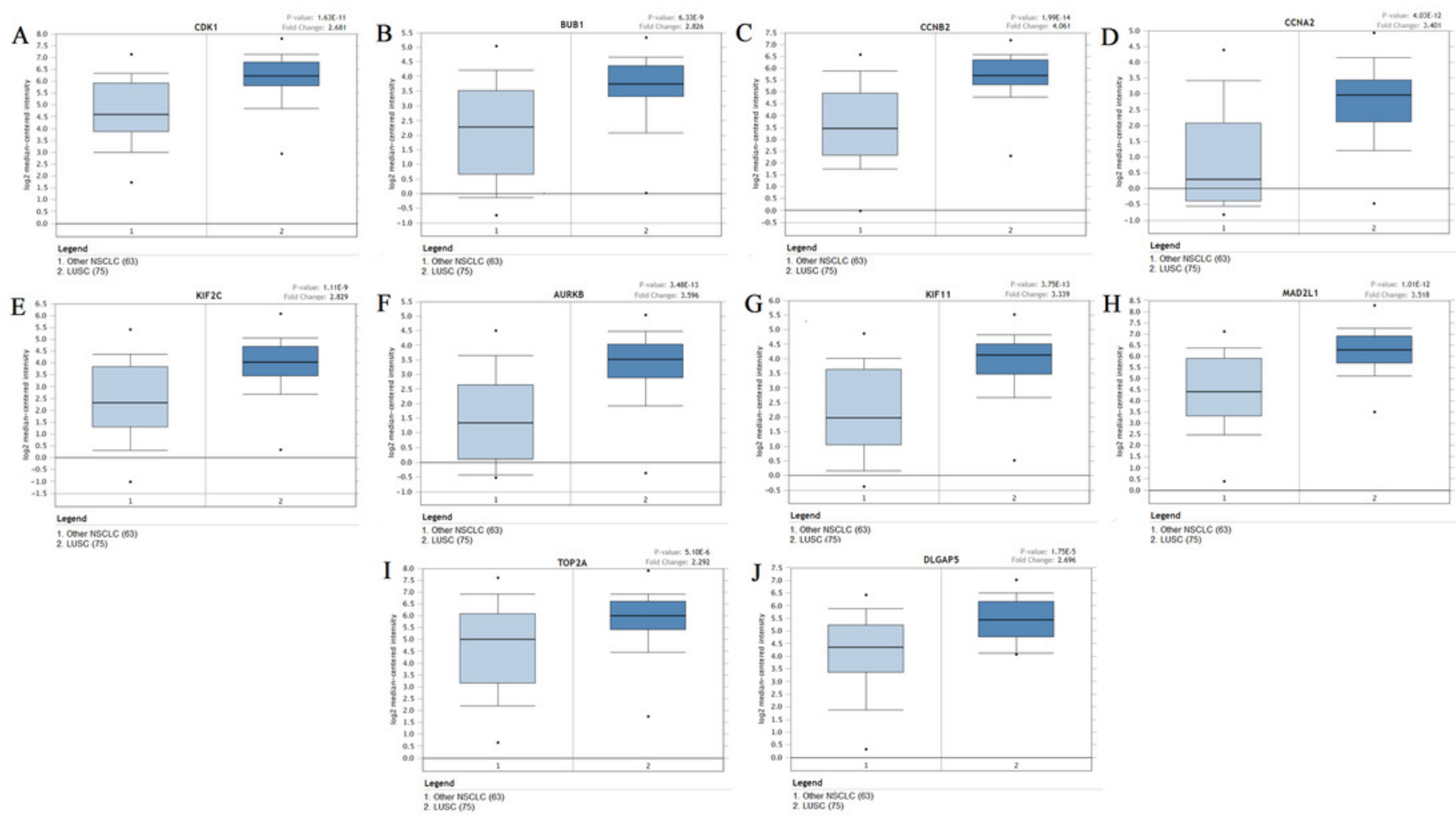

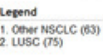


Figure 7

Kaplan-Meier survival analyses of hub genes in LUSC patients.

(A-D) The first progression survival analyses of the hub genes (CCNA2, KIF11, MAD2L1, and DLGAP5) were performed. (E) The post progression survival analyses of the hub gene (KIF2C) were performed. Logrank $P<0.05$ was considered statistically significant.
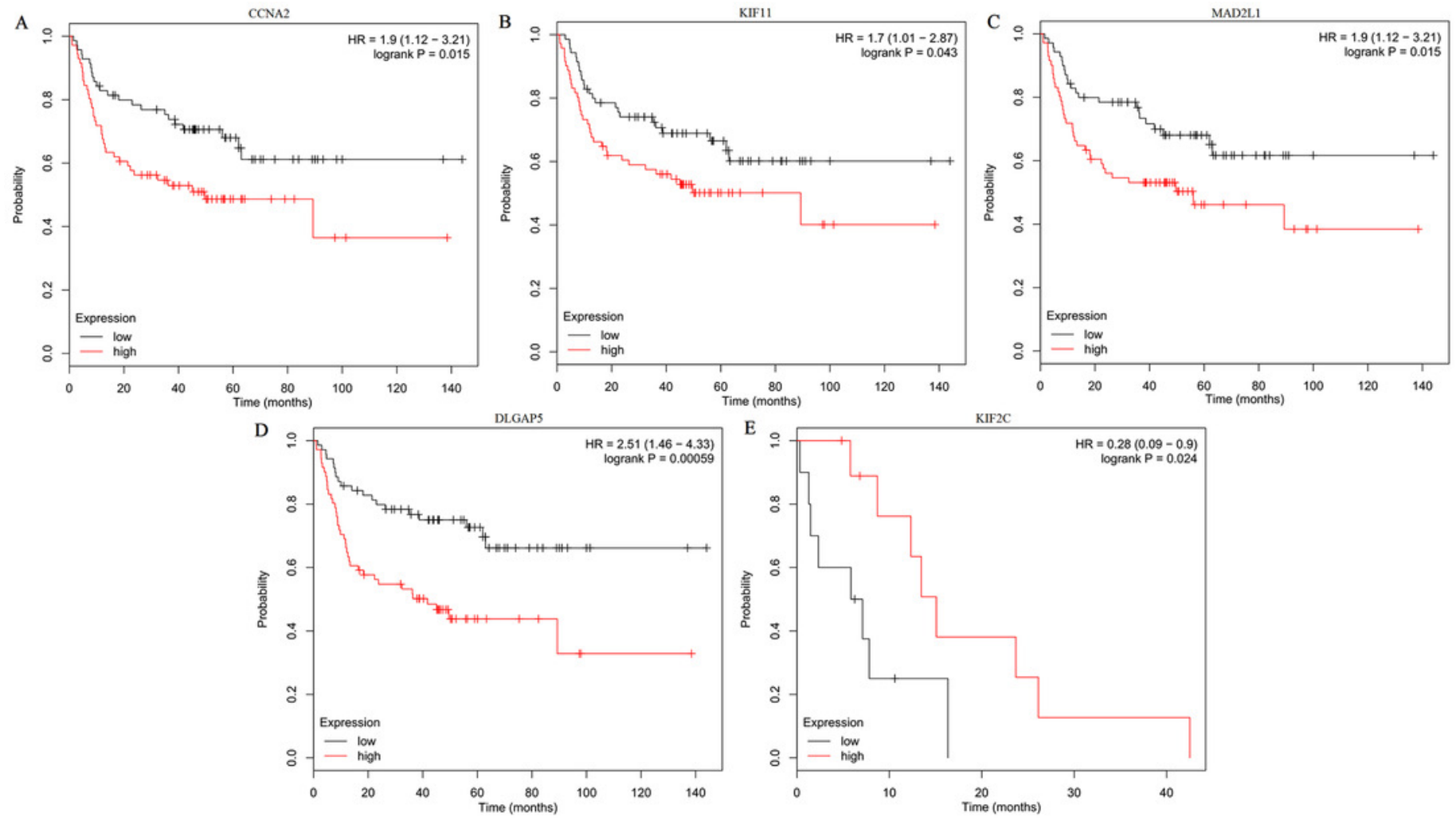
Figure 8

The association between the expression of prognosis-related hub genes and tumor stage (A-D).

In the Lee Lung dataset, the altered CCNA2, DLGAP5, MAD2L1 and KIF2C were associated with tumor grade in the LUSC progression. 0: No value; 1: Grade 1: high differentiation; 2: Grade 2: middle differentiation; Grade 3: poor differentiation.
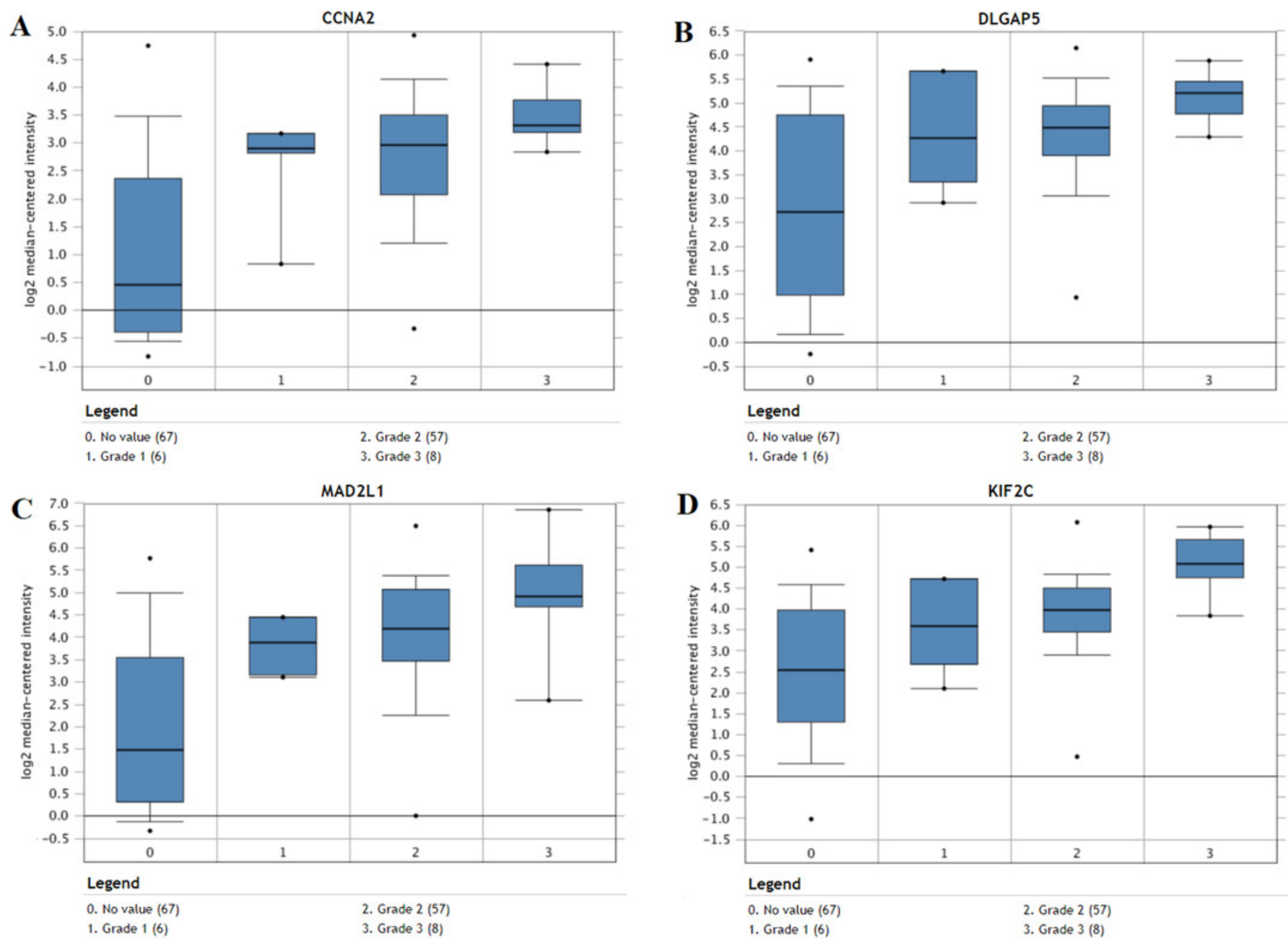
Figure 9

Prognosticgene signature of the seven genes in 488 LUSC patients.

(A) Risk score distribution. (B) Patients' survival status distribution. (C) Expression levels of the seven genes in low and high-risk groups (TCGA database).Gene expression values are log2-transformed. (D) The survival curves of LUSC patients in high- and low-risk groups. (E) time-dependent ROC curves for predicting OS in LUSC patients by the risk score.
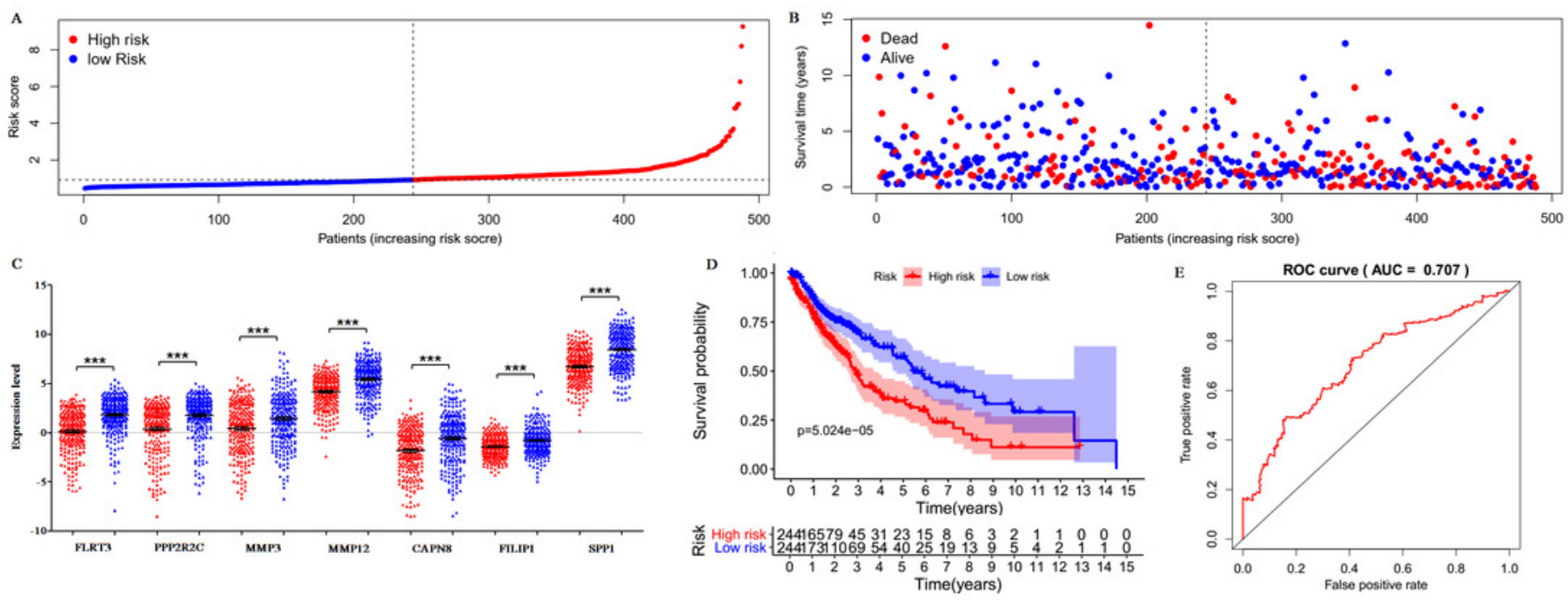